\title{
Comparação entre métodos de estimativa de evapotranspiração de referência no município de São José dos Ausentes (RS), Brasil
}

\author{
Comparison between methods of estimation of reference evapotranspiration \\ in the municipality of São José dos Ausentes (RS), Brazil
}

\author{
Julia Mattia Ongaratto* ${ }^{\circledR}$, Taison Anderson Bortolin' ${ }^{\circledR}$
}

\begin{abstract}
RESUMO
A evapotranspiração de referência é uma componente muito importante do balanço hídrico esua estimativa é essencial para execução de projetos agrícolas e ambientais, estudos de balanço hídrico, projetos e manejo de irrigação, modelagem de processos climatológicos e planejamento do gerenciamento dos recursos hídricos. O método de Penman-Monteith é considerado pela Food and Agriculture Organization of the United Nations como padrão para estimar a evapotranspiração de referência, contudo, dada a dificuldade de se obter um número grande de variáveis meteorológicas que são empregadas nesse método, tem-se utilizado vários outros métodos para estimar a evapotranspiração de referência. O presente trabalho teve como objetivo comparar, por meio de valores diários e mensais estimados pelo método de Penman-Monteith, o desempenho dos métodos de Thornthwaite, HargravesSamani, Makkink, Blaney-Criddle, Camargo e Jensen-Haise para o município de São José dos Ausentes, no Rio Grande do Sul. Os dados utilizados para estimar a evapotranspiração de referência foram obtidos pelo sistema Agritempo, que armazena e disponibiliza os dados da estação meteorológica automática do Instituto Nacional de Meteorologia. Os resultados apontam que o método de Blaney-Criddle foi o que apresentou os melhores resultados nas escalas tanto diária quanto mensal, seguido pelo método de Jensen-Haise, na escala mensal. Já os métodos que apresentaram os piores desempenhos foram o de Thorntwaite e Camargo, sendo classificados com desempenho "sofrivel" na escala mensal e como "péssimo" e "mau”, respectivamente, na escala diária.
\end{abstract}

Palavras-chave: Penman-Monteith; métodos indiretos; radiação solar.

\begin{abstract}
Reference evapotranspiration is a very important component of the water balance and its estimation is essential for the execution of agricultural and environmental projects, for studies of water balance, irrigation projects and management, modeling of climatological processes and planning of water resources management. The Penman-Monteith method is considered by the Food and Agriculture Organization of the United Nations as a standard for estimating reference evapotranspiration; however, due to the difficulty of obtaining a large number of meteorological variables that are employed in this method, several other methods have been used to estimate the reference evapotranspiration. The present coursework had the objective to compare the performance of the Thornthwaite, Hargraves-Samani, Makkink, Blaney-Criddle, Camargo, and Jensen-Haise methods for the municipality of São José dos Ausentes, using daily and monthly values estimated by the Penman-Monteith method. The data used to estimate the reference evapotranspiration were obtained through the Agritempo system, which stores and makes available the data of the automatic meteorological station of the National Meteorological Institute. The results show that the BlaneyCriddle method presented the best results, both daily and monthly, followed by the Jensen-Haise method in the monthly scale. On the other hand, the methods that presented the worst performances were those of Thorntwaite and Camargo, classified as "poor" on the monthly scale, and as "terrible" and "bad", respectively, on the daily one.
\end{abstract}

Keywords: Penman-Monteith; indirect methods; solar radiation.

\section{INTRODUÇÃO}

Dada a grande preocupação mundial com o uso racional da água, e tendo em vista que a água é um recurso natural de extrema necessidade para o homem, é muito importante o conhecimento acerca dos componentes do ciclo hidrológico, entre os quais, a evapotranspiração destaca-se por sua importância em abastecer a atmosfera de água (SILVEIRA \& STONE, 1994), que posteriormente contribuirá para a ocorrência de chuvas a nível continental. A estimativa da evapotranspiração de referência (ETo) auxilia pesquisadores e profissionais da área na execução de projetos agrícolas e ambientais e é necessária para estudos de balanço hídrico, projetos e manejo de irrigação,

UUniversidade de Caxias do Sul - Caxias do Sul (RS), Brasil.

*Autor correspondente: julia_mattia@hotmail.com

Conflitos de interesse: os autores declaram não haver conflitos de interesse

Financiamento: nenhum.

Recebido: 23/06/2019 - Aceito: 06/11/2020 - Reg. ABES: 20190196 
modelagem de processos climatológicos e planejamento do gerenciamento dos recursos hídricos.

Em função da dificuldade para realizar as medições diretas, a aplicação dos métodos indiretos tem-se tornado uma ferramenta em potencial para determinar a evapotranspiração, tal como apresentado nos trabalhos de Sanches et al. (2015), Pilau et al. (2012) e Paiva et al. (2016). Os métodos indiretos são utilizados para estimar a transferência de vapor d'água para a atmosfera em condições climáticas específicas, contudo poucos têm sido adaptados para situações climáticas distintas daquelas que lhes deram origem (VAREJÃO-SILVA, 2006).

Entre os diversos métodos de cálculo da evapotranspiração, destaca-se o método de Penman-Monteith, recomendado pela Food and Agriculture Organization of the United Nations (FAO) e utilizado para a calibração de outros métodos, visto que este se mostra eficiente em diversas condições climáticas. Camargo e Camargo (2000) enfatizam que, em alguns casos, o uso do método de Penman-Monteith é restrito pela falta de algumas variáveis meteorológicas, o que gera a necessidade da utilização de outros métodos indiretos, menos exigentes em relação aos dados requeridos.

Ante o que foi exposto, o objetivo deste trabalho foi avaliar o desempenho dos métodos indiretos para estimar a ETo, tais como: Thornthwaite, HargravesSamani, Makkink, Blaney-Criddle, Camargo e Jensen-Haise, nas escalas diárias e mensais, comparando com o método padrão de Penman-Monteith FAO, no município de São José dos Ausentes (RS).

\section{METODOLOGIA}

Os dados meteorológicos utilizados neste estudo correspondem ao período de janeiro de 2008 a dezembro de 2010 obtidos por meio do sistema Agritempo e calculados os valores de evapotranspiração com uma aplicação web elaborada por Bortolin et al. (2018) que utiliza os dados do Instituto Nacional de Meteorologia (INMET), especificamente da estação meteorológica situada em São José dos Ausentes (RS) (Figura 1), com coordenadas geográficas de $-28.748615^{\circ}$ de latitude e $-50.057869^{\circ}$ de longitude, com altitude de $1.229 \mathrm{~m}$. O clima da região é classificado, segundo Koppen, como do tipo Cfa, que se caracteriza por apresentar chuvas durante todos os meses do ano e possuir a temperatura do mês mais quente superior a $22^{\circ} \mathrm{C}$ e a do mês mais frio superior a $3^{\circ} \mathrm{C}$. A precipitação pluviométrica total anual pode variar de 1.400 a $1.700 \mathrm{~mm}$.

Os dados dessa estação foram utilizados para o cálculo da evapotranspiração de referência por meio de sete métodos indiretos, nas escalas de tempo diária e mensal, tomando como estimativa padrão o método de Penman-Monteith. Os demais métodos utilizados foram: Thornthwaite, Hargraves-Samani, Makkink, Blaney-Criddle, Camargo e Jensen-Haise.

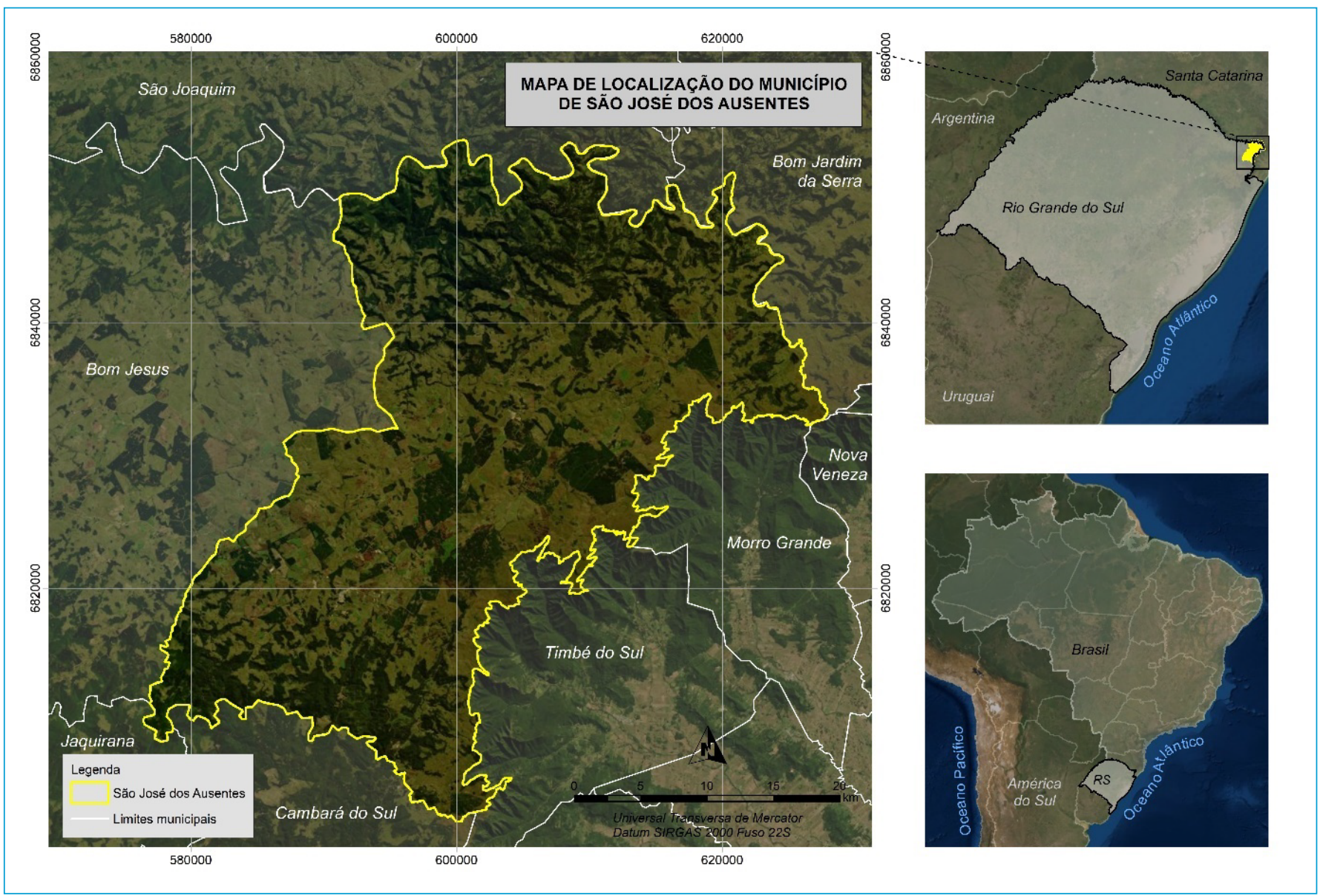

Figura 1 - Localização do município. 


\section{Método de Penman-Monteith FAO-56}

O método de Penman-Monteith FAO-56 (ALLEN et al., 1998), obtido por meio de diversas parametrizações, é dado pela Equação 1.

ETo $(P M)=\frac{0,408 \cdot \Delta\left(R_{n}-G\right)+\gamma \cdot \frac{900}{\left(T_{\text {med }}+273\right)} \cdot U_{2}\left(e_{s}-e_{s}\right)}{\Delta+\gamma \cdot\left(1+0,34 U_{2}\right)}$

Em que:

ETo (PM): evapotranspiração de referência estimada pelo método de PenmanMonteith FAO-56 (mm.dia $\left.{ }^{-1}\right)$;

$\Delta$ : declividade da curva de pressão de vapor na saturação $\left(\mathrm{kPa}^{\circ} \mathrm{C}^{-1}\right)$;

$\mathrm{R}_{\mathrm{n}}$ : radiação líquida $\left(\mathrm{MJ} \mathrm{m}^{-2} \mathrm{dia}^{-1}\right)$;

G: radiação de calor no solo $\left(\mathrm{MJ} \mathrm{m}^{-2} \mathrm{dia}^{-1}\right)$;

$\gamma$ : constante psicrométrica $\left(\mathrm{kPa}^{\circ} \mathrm{C}^{-1}\right)$;

$\mathrm{T}_{\text {med }}$ : temperatura média diária $\left({ }^{\circ} \mathrm{C}\right)$;

$\mathrm{U}_{2}$ : velocidade do vento a 2 metros de altura $\left(\mathrm{m} . \mathrm{s}^{-1}\right)$;

$\mathrm{e}_{\mathrm{s}}$ : pressão de vapor na saturação $(\mathrm{kPa})$;

$\mathrm{e}_{\mathrm{a}}$ : pressão de vapor atual $(\mathrm{kPa})$.

\section{Método de Thornthwaite}

O método de Thornthwaite foi obtido por meio das Equações 2, 3 e 4 .

ETo $(\mathrm{TW})=16\left(10 \frac{\mathrm{Ti}}{\mathrm{I}}\right)^{\mathrm{a}}$

$\mathrm{a}=6,75 \cdot 10^{-7} \mathrm{I}^{3}-7,71 \cdot 10^{-5} \mathrm{I}^{2}+1,7912 \cdot 10^{-2} \mathrm{I}+0,49239$

$\mathrm{I}=\sum_{\mathrm{i}=1}^{12}(0,2 \mathrm{Ti})^{1,514}$

Em que:

ETo (TW): evapotranspiração de referência estimada pelo método de Thornthwaite (mm.mês $\left.{ }^{-1}\right)$;

Ti: temperatura média mensal $\left({ }^{\circ} \mathrm{C}\right)$;

a: expoente função do índice anual I;

i: índice mensal de calor para o mês j;

I: índice anual, que corresponde ao somatório dos 12 índices i mensais.

\section{Método de Hargreaves-Samani}

O método de Hargreaves-Samani foi obtido por meio da Equação 5.

ETo (HG) - 0,0023 ( $\left.\mathrm{T}_{\text {med }}+17,8\right) \cdot\left(\mathrm{T}_{\max }-\mathrm{T}_{\min }\right)^{0,5} \cdot \mathrm{Ra}$

Em que:

ETo (HG): evapotranspiração de referência estimada pelo método de HargreavesSamani $\left(\mathrm{mm} \cdot \mathrm{dia}^{-1}\right)$;

$\mathrm{T}_{\text {med }}$ : temperatura média do ar diária $\left({ }^{\circ} \mathrm{C}\right)$;

$\mathrm{T}_{\max }$ : temperatura máxima do ar diária $\left({ }^{\circ} \mathrm{C}\right)$;

$\mathrm{T}_{\min }$ : temperatura mínima do ar diária $\left({ }^{\circ} \mathrm{C}\right)$;

Ra: radiação solar extraterrestre $\left(\mathrm{MJm}^{2} \mathrm{dia}^{-1}\right)$.

\section{Método de Makkink}

Para calcular a evapotranspiração pelo método de Makkink, utilizaram-se as Equações 6 e 7.
$\operatorname{ETo}(M K)=0,61 \cdot W \cdot \operatorname{Rs}_{(d)}-0,12$

$W=\frac{\Delta}{\Delta+Y}$

Em que:

ETo (MK): evapotranspiração de referência estimada pelo método de Makkink $\left(\mathrm{mm} \cdot \mathrm{dia}^{-1}\right)$;

$\mathrm{Rs}_{(\mathrm{d})}$ : radiação solar incidente $\left(\mathrm{mm} \cdot \mathrm{dia}^{-1}\right)$;

W: fator de ponderação;

$\Delta$ : declividade da curva de pressão de vapor em relação à temperatura;

Y: constante psicométrica.

\section{Método de Blaney-Criddle}

Para calcular a evapotranspiração pelo método de Blaney-Criddle, utilizaram-se as Equações 8, 9, 10 e 11.

ETo $(B C)=a+b \cdot\left[P .\left(0,46 T_{\text {med }}+8,13\right)\right]$

$\mathrm{a}=0,0043 \mathrm{UR}_{\min }-\left(\frac{\mathrm{n}}{\mathrm{N}}\right)-1,41$

$\mathrm{b}=\mathrm{a}_{\mathrm{o}}+\mathrm{a}_{1} \cdot \mathrm{UR}_{\min }+\mathrm{a}_{2} \cdot \frac{\mathrm{n}}{\mathrm{N}}+\mathrm{a}_{3} \cdot \mathrm{U}_{\mathrm{d}}+\mathrm{c}$

$c=a_{4} \cdot U R_{\min } \cdot \frac{n}{N}+a_{5} \cdot U R_{\min } \cdot U_{d}$

Em que:

ETo (BC): evapotranspiração de referência estimada pelo método de Blaney-

Criddle ( $\left.\mathrm{mm} \cdot \mathrm{dia}^{-1}\right)$;

P: percentagem média diária de horas de luz para diferentes latitudes;

$\mathrm{T}_{\text {med }}$ : temperatura média diária do ar calculada pelas temperaturas máxima e mínima do ar $\left({ }^{\circ} \mathrm{C}\right)$;

$\mathrm{a}+\mathrm{b}$ : coeficientes regionais de ajuste da equação;

$\mathrm{a}_{\mathrm{o}}: 0,811917$

$a_{1}:(-0,0040922)$;

$\mathrm{a}_{2}: 1,0705$

$\mathrm{a}_{3}: 0,065649$;

$a_{4}:(-0,0059684)$

$\mathrm{a}_{5}:(-0,0005967)$;

$\mathrm{UR}_{\min }$ : umidade relativa mínima do ar (\%);

n: número de horas reais de insolação (h). Pela ausência de medições em campo, considerou-se a equação de Angstrom-Prescott para sua estimativa;

$\mathrm{N}$ : fotoperíodo (h);

$\mathrm{U}_{\mathrm{d}}$ : velocidade do vento do período diurno a $2 \mathrm{~m}$ de altura $\left(\mathrm{m} \cdot \mathrm{s}^{-1}\right)$.

\section{Método de Camargo}

Para calcular a evapotranspiração pelo método de Camargo, empregou-se a Equação 12.

ETo (C) - $\mathrm{R}_{\mathrm{a}(\mathrm{dia})} \cdot \mathrm{T} \cdot \mathrm{K}_{\mathrm{F}}$

Em que:

ETo (C): evapotranspiração de referência estimada pelo método de Camargo $\left(\mathrm{mm} \cdot \mathrm{dia}^{-1}\right)$; 
$\mathrm{R}_{\mathrm{a}(\mathrm{dia})}:$ radiação solar extraterrestre $\left(\mathrm{MJm}^{2} \mathrm{dia}^{-1}\right)$;

T: temperatura média diária do $\operatorname{ar}\left({ }^{\circ} \mathrm{C}\right)$;

$\mathrm{K}_{\mathrm{F}}$ : fator de ajuste que varia com a temperatura média anual do ar no local de estudo $\left(\mathrm{K}_{\mathrm{f}}=0,01\right.$ para $\left.\mathrm{T}<23^{\circ} \mathrm{C}\right)$;

$\mathrm{K}_{\mathrm{f}}=0,0105$ para $\mathrm{T}=24^{\circ} \mathrm{C}$;

$\mathrm{K}_{\mathrm{f}}=0,011$ para $\mathrm{T}=25^{\circ} \mathrm{C}$;

$\mathrm{K}_{\mathrm{f}}=0,0115$ para $\mathrm{T}=26^{\circ} \mathrm{C}$;

$\mathrm{K}_{\mathrm{f}}=0,012$ para $\mathrm{T}>26^{\circ} \mathrm{C}$ ).

\section{Método de Jensen-Haise}

As equações utilizadas para o cálculo da evapotranspiração pelo método de Jensen-Haise foram as Equações 13 e 14.

ETo $(J H)=\mathrm{Rs}_{(\mathrm{d})} \cdot(0,0252 \mathrm{~T}=0,078)$

$\operatorname{Rs}(\mathrm{mm})=\frac{\mathrm{Rs}}{\lambda}$

Em que:

ETo (JH): evapotranspiração de referência estimada pelo método de JensenHaise $\left(\mathrm{mm} \cdot \mathrm{dia}^{-1}\right)$;

$\mathrm{Rs}_{(\mathrm{d})}$ : radiação solar incidente $\left(\mathrm{MJm}^{2} \mathrm{dia}^{-1}\right)$;

$\mathrm{T}$ : temperatura média diária do $\operatorname{ar}\left({ }^{\circ} \mathrm{C}\right)$;

Rs $(\mathrm{mm})$ : radiação solar incidente $\left(\mathrm{mm} \cdot \mathrm{dia}^{-1}\right)$;

$\lambda$ : calor latente de vaporização $\left(\mathrm{MJ} \cdot \mathrm{mm}^{-1}\right)$.

\section{Comparação dos métodos}

A comparação dos resultados foi executada pelos valores médios mensais da série histórica e também pela análise do desempenho dos métodos de estimativa de ETo em relação à equação padrão ETo (PM) para a escala diária e mensal. Realizou-se a análise de correlação e de regressão linear por meio da geração de gráficos diários e mensais, por meio dos quais foram obtidos os coeficientes linear (a) e angular (b) da equação do tipo $y=a x+b$, em que $y$ é o método padrão de estimativa (ETo (PM), mm.d $\mathrm{d}^{-1}$ e $x$ é a ETo estimada por meio de outro método $\left(\mathrm{mm} \cdot \mathrm{d}^{-1}\right)$.

Também se obtiveram o coeficiente de determinação da regressão linear $\left(r^{2}\right)$, o coeficiente de correlação $(r)$ e o índice de concordância $(d)$ de Willmott et al. (1985) que foram calculados pelas Equações 15, 16 e 17, respectivamente. Todos os cálculos foram realizados com o uso da ferramenta Excel ${ }^{\circledR}$.

$r^{2}=\frac{\left[\sum_{i=1}^{N}(P i-\bar{P}) \cdot(O i-\bar{O})\right]^{2}}{\sum_{i=1}^{N}(P i-\bar{P})^{2} \cdot \sum_{i=1}^{N}(O i-\bar{O})^{2}}$

$r=\frac{\sum_{i=1}^{N}(P i-\bar{P}) \cdot(O i-\bar{O})}{\sqrt{\sum_{i=1}^{N}(P i-\bar{P})^{2} \cdot \sum_{i=1}^{N}(O i-\bar{O})^{2}}}$

$d=1-\frac{\sum_{i=1}^{N}(P i-O i)^{2}}{\sum_{i=1}^{N}(|P i-\bar{O}|+|O i-\bar{O}|)^{2}}$

Em que:

$P i$ : são os valores estimados pelos métodos propostos;

$\bar{P}$ : média dos valores estimados pelos métodos propostos;

Oi: são os valores estimados pelo Método Penman-Monteith Padrão FAO

(ETo (PM));
$\bar{O}:$ média dos valores estimados pelo Método Penman-Monteith Padrão FAO (ETo (PM)).

O índice de concordância de Wilmott varia de 0 (total discordância) a 1 (concordância perfeita).

Com base no cálculo de $d$ e $r$, é calculado o índice de desempenho proposto por Camargo e Sentelhas (1997), denominado de índice de desempenho, dado pela Equação 18.

$c=r \cdot d$

Com base nos resultados para esse índice, os autores propuseram uma tabela de avaliação qualitativa (Tabela 1). Os parâmetros de comparação foram calculados para as escalas diária e mensal.

\section{RESULTADOS E DISCUSSÃO}

Na Tabela 2 e na Figura 2 são apresentadas as médias mensais da ETo ao longo dos três anos de cálculo, obtidas por meio dos métodos analisados na área de estudo.

Observa-se que os meses que apresentaram a maior ETo estimada pelo método de Penman-Monteith foram os de dezembro, janeiro e fevereiro, meses nos quais são registradas temperaturas mais elevadas em decorrência do maior índice de radiação solar, contribuindo para o acréscimo de uma demanda evaporativa do ar (Figura 2). Encontraram-se valores superiores a $100 \mathrm{~mm} . \mathrm{mês}^{-1}$, enquanto o mês de junho apresentou os menores valores para todos os métodos, quando se dá início ao período de inverno na Região Sul, e, neste mês, a média entre os métodos utilizados foi de 41,16 mm, com desvio padrão de 13,09 mm.

O método de Blaney-Criddle superestimou a ETo em comparação ao Penman-Monteith em todos os meses do ano, com diferenças que variaram entre $10 \%$ no mês de junho e $19 \%$ no mês de dezembro. Fernandes (2006) e Back (2008) também encontraram resultados semelhantes: os dois constataram tendência à superestimativa do método de Blaney-Criddle ao longo de todo o ano.

Nos meses em que ocorrem as maiores temperaturas, como nas estações de primavera e verão, o método de Hargreaves-Samani superou os valores de Penman-Monteith. Esse método se mostra uma boa alternativa em regiões onde a disponibilidade de dados é limitada, pois depende apenas da temperatura e radiação, porém, conforme Lima Junior et al. (2016), ele superestima a equação modelo nos primeiros meses do ano, como também observado neste estudo.

Tabela 1 - Índice de concordância.

\begin{tabular}{l|c} 
Valor de "c" & Desempenho \\
$>0,85$ & Ótimo \\
\hline $0,76-0,85$ & Muito bom \\
\hline $0,66-0,75$ & Bom \\
\hline $0,61-0,65$ & Mediano \\
\hline $0,51-0,6$ & Sofrível \\
\hline $0,41-0,5$ & Mau \\
\hline$\leq 0,40$ & Péssimo \\
\hline
\end{tabular}

c: índice de concordância.

Fonte: Camargo e Sentelhas (1997). 
Tabela 2 - Resultados médios mensais de estimativa da evapotranspiração de referência (mm.mês'-1) para os métodos utilizados.

\begin{tabular}{|c|c|c|c|c|c|c|c|c|c|}
\hline $\begin{array}{l}\text { Mês / } \\
\text { Método }\end{array}$ & Blaney-Criddle & Camargo & $\begin{array}{l}\text { Hargreaves- } \\
\text {-Samani }\end{array}$ & $\begin{array}{l}\text { Jensen- } \\
\text {-Haise }\end{array}$ & Makkink & $\begin{array}{l}\text { Penman- } \\
\text {-Monteith }\end{array}$ & Thornthwaite & Média & $\begin{array}{l}\text { Desvio } \\
\text { Padrão }\end{array}$ \\
\hline Janeiro & 125,86 & 94,73 & 119,21 & 130,31 & 99,86 & 105,86 & 87,90 & 109,10 & 16,25 \\
\hline Fevereiro & 123,56 & 85,71 & 109,17 & 126,39 & 94,03 & 105,65 & 84,07 & 104,08 & 17,05 \\
\hline Março & 116,89 & 76,02 & 97,87 & 116,65 & 89,36 & 100,30 & 78,62 & 96,53 & 16,47 \\
\hline Abril & 100,26 & 49,93 & 72,64 & 81,45 & 66,92 & 82,98 & 55,57 & 72,82 & 17,26 \\
\hline Maio & 76,18 & 33,51 & 53,14 & 54,85 & 49,00 & 63,17 & 40,64 & 52,93 & 14,10 \\
\hline Junho & 62,06 & 23,14 & 41,54 & 38,42 & 38,44 & 56,07 & 28,48 & 41,16 & 13,90 \\
\hline Julho & 85,76 & 28,27 & 50,03 & 50,04 & 47,29 & 72,09 & 35,30 & 52,68 & 20,05 \\
\hline Agosto & 99,65 & 38,12 & 64,72 & 66,01 & 58,68 & 86,83 & 42,65 & 65,24 & 22,14 \\
\hline Setembro & 85,46 & 45,12 & 74,53 & 63,43 & 59,60 & 72,08 & 42,39 & 63,23 & 15,68 \\
\hline Outubro & 107,87 & 64,26 & 99,33 & 94,68 & 82,60 & 90,23 & 57,07 & 85,15 & 18,55 \\
\hline Novembro & 124,75 & 83,71 & 114,72 & 119,57 & 94,87 & 104,41 & 76,58 & 102,66 & 18,36 \\
\hline Dezembro & 148,10 & 94,37 & 127,17 & 143,54 & 111,87 & 120,20 & 86,72 & 118,85 & 23,15 \\
\hline Média & 104,70 & 59,74 & 85,34 & 90,45 & 74,38 & 88,32 & 59,67 & & \\
\hline Desvio Padrão & 24,59 & 26,55 & 29,52 & 36,01 & 24,01 & 19,68 & 21,97 & & \\
\hline
\end{tabular}

Fonte: Elaboração própria.

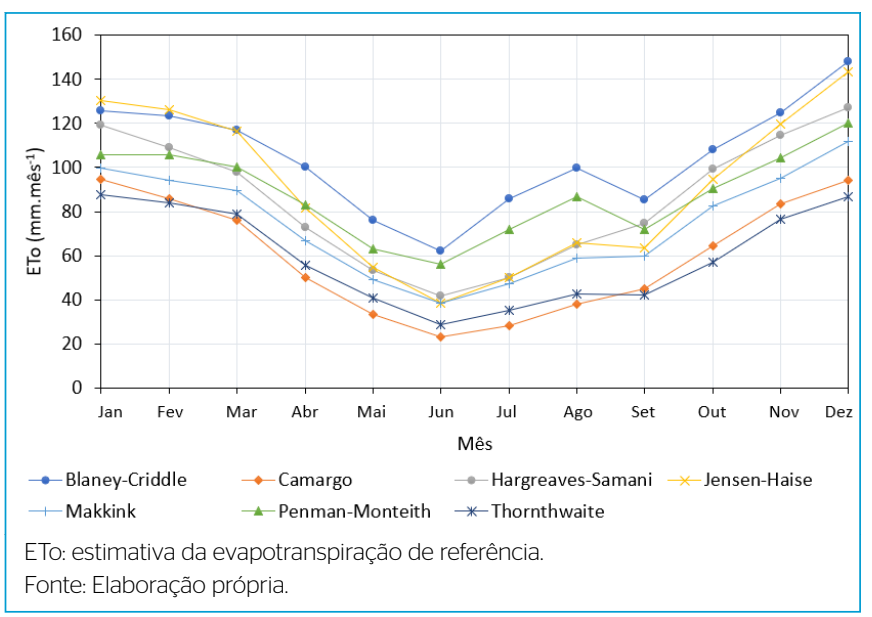

Figura 2 - Médias mensais da estimativa da evapotranspiração de referência.

O mesmo ocorreu com o método de Jensen-Haise, que também superestimou a ETo entre os meses de outubro e março, com diferenças entre 5\% (outubro) e 19\% (janeiro). Já os métodos de Thornthwaite, Makkink e Camargo apresentaram a tendência de subestimar a ETo em todos os meses do ano. Borges e Mendiondo (2007) afirmam que o método de Thornthwaite possui a tendência a mais erros nos meses mais secos e menos erros nos meses chuvosos. Camargo et al. (1999) comentam que o método original de Thornthwaite não estima satisfatoriamente a evapotranspiração, por não considerar o termo aerodinâmico ou a contribuição da energia latente, e seu resultado pode ter sido reflexo da ausência desse termo em sua formulação. Além disso, cabe destacar que o método de Thornthwaite, diferentemente dos outros métodos, é calculado mensalmente e, por isso, é um método que se torna pouco preciso para uso em tomadas de decisão em curto prazo (CRUZ, 2016).
Os valores diários e mensais estimados de ETo para os diferentes métodos são apresentados respectivamente nas Figuras 3 e 4.

Na escala diária, observa-se que os métodos de Camargo e de Thornthwaite foram os que ficaram mais distantes dos valores estimados por PenmanMonteith, apresentando um valor de $\mathrm{r}^{2}$ igual a 0,376 e 0,449, respectivamente. A semelhança entre os dois é esperada, já que o método de Camargo foi originado do método de Thornthwaite. Ambos utilizam dados de temperatura de entrada, no entanto Camargo considera um fator de ajuste que varia conforme a temperatura média anual do ar no local de estudo, o que é apontado como o motivo de o método de Camargo apresentar resultados um pouco melhores que Thornthwaite. Quando os valores encontrados para $\mathrm{r}^{2}$ estão muito baixos, os métodos não se mostram uma boa opção para serem aplicados e há a necessidade de ajustes locais para que a fórmula seja mais eficiente na estimativa de ETo (PALARETTI et al., 2014).

Analisando a Figura 4, assim como o que ocorre na escala diária, na escala mensal os métodos de Camargo e de Thornthwaite ficaram mais distantes dos valores estimados por Penman-Monteith, apresentando um valor de $\mathrm{r}^{2}$ igual a 0,7730 e 0,7705 , respectivamente.

A Tabela 3 mostra os resultados dos índices e coeficientes calculados, além do desempenho de cada método.

Os resultados apresentados na Tabela 3 permitem identificar que, entre os seis métodos analisados, o que apresentou melhor desempenho em relação a Penman-Monteith foi o método de Blaney-Criddle, com desempenho "ótimo" nas escalas diária e mensal com índice de concordância "c" igual a 0,87 e 0,93, respectivamente. Gotardo et al. (2016) recomendam a utilização desse método quando houver ausência de longas séries históricas de dados climatológicos que impossibilitem o uso do método padrão de Penman-Monteith, apesar de que a aplicação do método é mais complicada que a de outros métodos que são baseados em temperatura, como Camargo e Hargreaves-Samani (CAMARGO \& SENTELHAS, 1997; BORGES JUNIOR et al., 2012). 

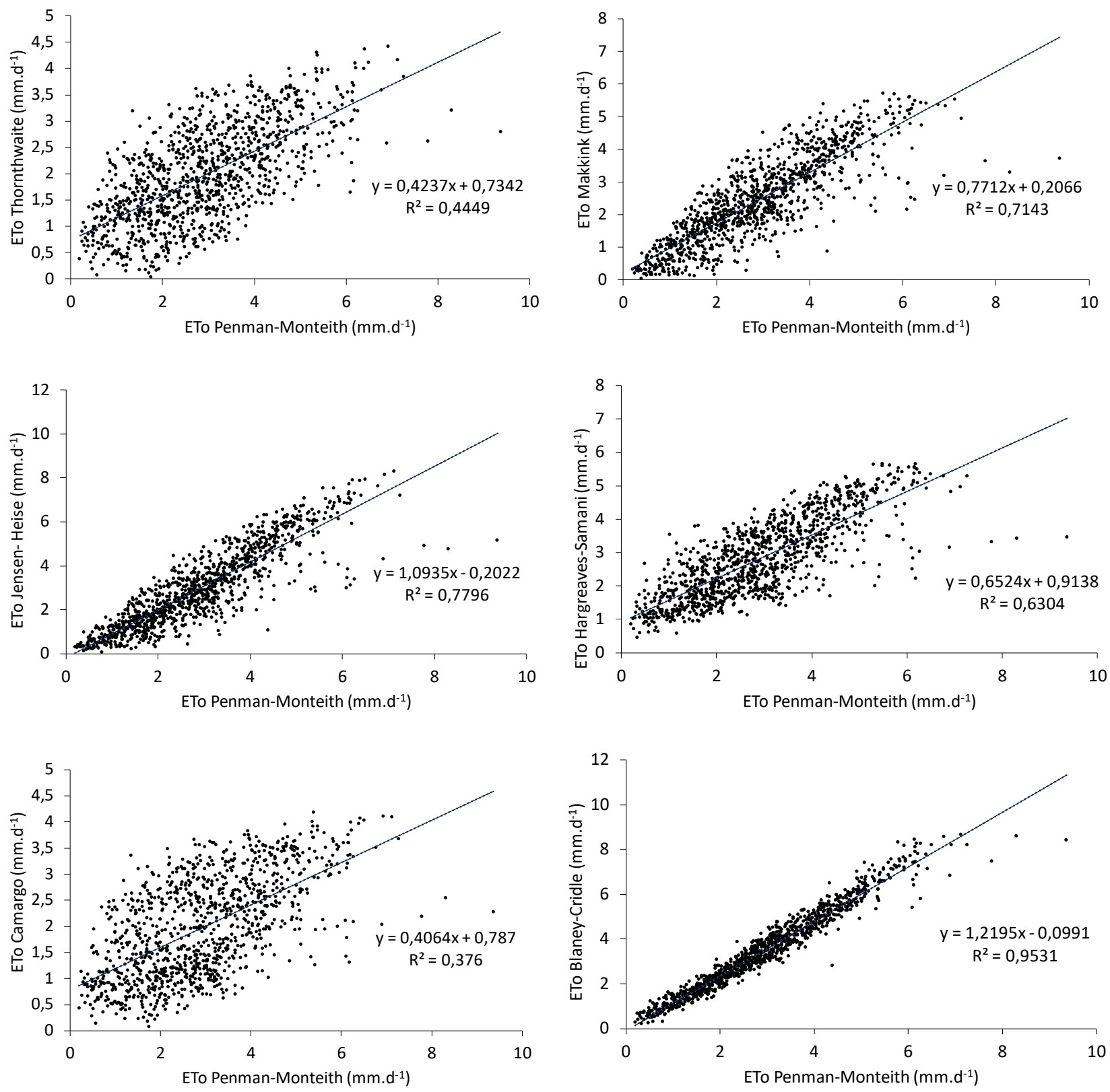

ETo: estimativa da evapotranspiração de referência.

Fonte: Elaboração própria.

Figura 3 - Correlações entre os valores de estimativa da evapotranspiração de referência observados e estimados pelos métodos avaliados no período em estudo na escala diária.

O método de Jensen-Haise também foi classificado com desempenho "ótimo", apresentando índice "c" igual a 0,86 na escala mensal, no entanto o desempenho do método diminui na avaliação em escala diária, sendo classificado como "muito bom", com valor do índice "c" igual a 0,81. Brixner et al. (2014), em estudo realizado na Campanha Gaúcha, constatou que, entre todos os métodos analisados, o que teve o melhor desempenho foi o de Jensen-Haise, com "c" igual a 0,78 , sendo classificado como "muito bom". Segundo Jung et al. (2016), o método de Jensen-Haise apresenta um desempenho bom no período de secas, porém inferior no período chuvoso.
Em estudo realizado por Pereira et al. (2009) na região da Serra da Mantiqueira, que também possui invernos frios com verões com temperaturas amenas e mais chuvosos, os métodos de Jensen-Haise e Blaney-Criddle também tiveram os melhores desempenhos.

O método de Hargreaves-Samani apresentou desempenho "muito bom" na escala mensal, com valor de "c" igual 0,85 e "d" igual a 0,93, no entanto, na escala diária, o seu desempenho caiu, sendo classificado como "bom", com coeficiente "c" igual a 0,69. Conceição e Mandelli (2005) e Gonçalves et al. (2009) também encontraram resultados semelhantes, ambos classificando o método 

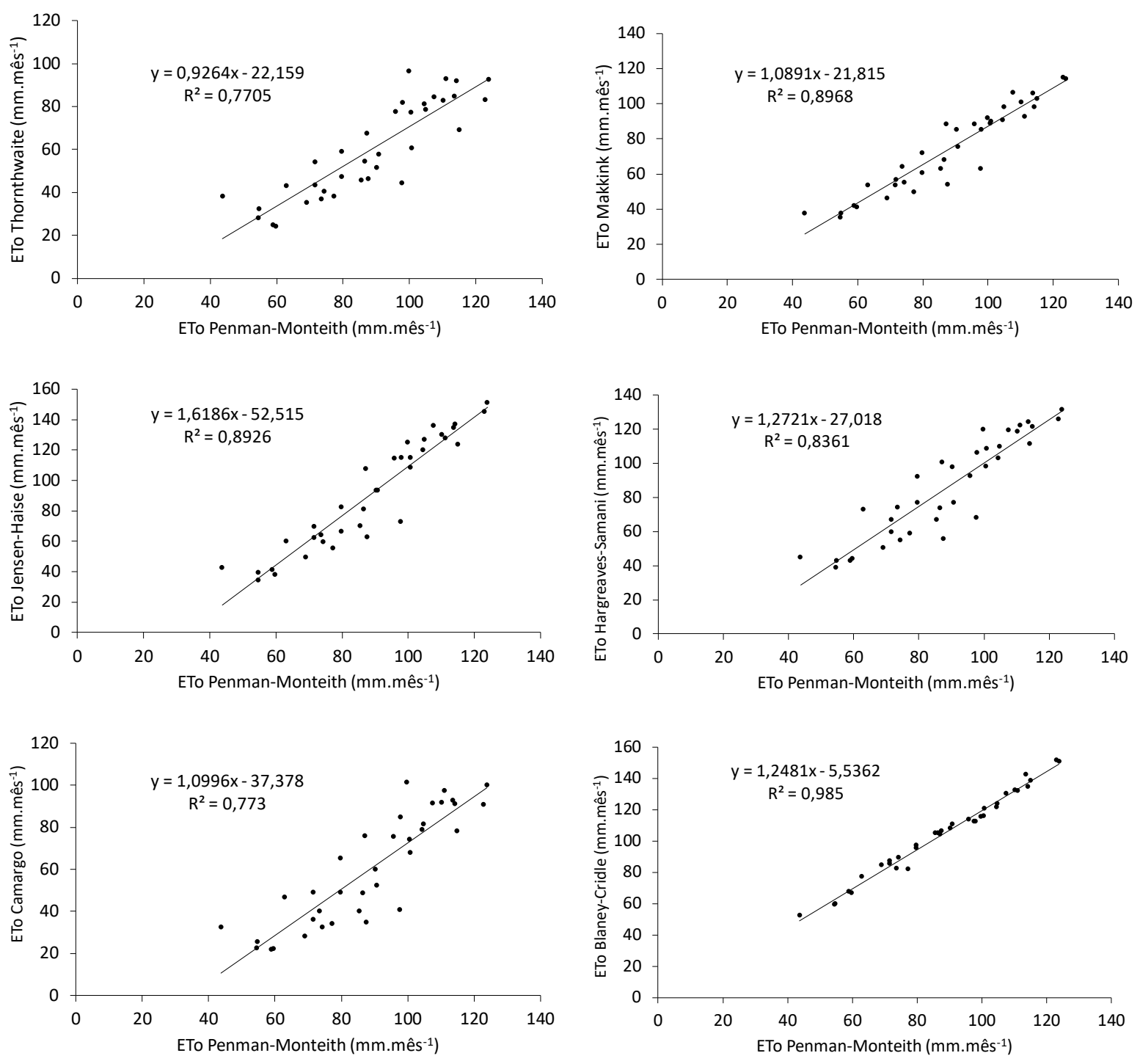

ETo: estimativa da evapotranspiração de referência.

Fonte: Elaboração própria.

Figura 4 - Correlações entre os valores de estimativa da evapotranspiração de referência observados e estimados pelos métodos avaliados no período em estudo na escala mensal.

com desempenho "muito bom" na escala diária. Pelo fato de esse método utilizar somente dados de temperatura (máxima e mínima) e latitude local, que possui relação com a radiação extraterrestre e varia em função dos dias, pode ser utilizado facilmente, mesmo que não haja dados de radiação solar da região (JUNIOR et al., 2012).

O método de Makkink, que utiliza como variável de entrada a temperatura do ar e a radiação solar global, apresentou desempenho "muito bom", com valores de "c" igual a 0,83 na escala mensal. Porém, quando analisado em escala diária, o seu desempenho cai, sendo classificado como "bom".

Entre os métodos analisados, os que apresentaram os piores resultados foram Thornthwaite seguido por Camargo, ambos classificados como "sofrível” na escala mensal. Quando a avaliação é feita diariamente, o desempenho é ainda pior, sendo classificado como "mau" para Thornthwaite e "péssimo" para Camargo. Apesar de o modelo de Thornthwaite funcionar bem em climas úmidos (CAMARGO \& CAMARGO, 2000), ainda que corrigidos pela temperatura efetiva do método de Camargo, esses métodos apresentaram resultados menos satisfatórios do que aqueles que se baseiam na radiação solar. O método de Camargo teve índice "c" igual a 0,59 na escala mensal e 0,40 na escala diária, sendo classificado como "sofrível" e "mau", respectivamente. Oliveira et al. (2008) também encontraram um desempenho "sofrível" para o método, com valor de "c" igual a 0,53 .

Os resultados encontrados neste trabalho corroboram os dados de Gonçalves et al. (2009), Cavalcanti Junior et al. (2010) e Almeida et al. (2010), os quais mostram que os métodos baseados na radiação solar apresentaram bons resultados 
Tabela 3 - Índice estatístico de comparação entre os métodos para a área de estudo nas escalas mensal e diária.

\begin{tabular}{l|c|c|c|c|c|c}
\multirow{2}{*}{ Blaney-Criddle } & $r^{2}$ & $r$ & $d$ & $c$ & \multirow{2}{*}{ Desempenho } \\
\cline { 2 - 5 } & \multicolumn{5}{|c|}{ Mensal } \\
\hline Camargo & 0,98 & 0,99 & 0,87 & 0,87 & Ótimo \\
\hline Hargreaves-Samani & 0,77 & 0,88 & 0,68 & 0,59 & Sofrível \\
\hline Jensen-Haise & 0,84 & 0,91 & 0,93 & 0,85 & Muito bom \\
\hline Makkink & 0,89 & 0,94 & 0,90 & 0,86 & Ótimo \\
\hline Thornthwaite & 0,89 & 0,95 & 0,88 & 0,83 & Muito bom \\
\hline Blaney-Criddle & 0,77 & 0,88 & 0,66 & 0,58 & Sofrível \\
\hline Camargo & 0,95 & 0,98 & 0,95 & 0,93 & Ótimo \\
\hline Hargreaves-Samani & 0,63 & 0,79 & 0,88 & 0,69 & Péssimo \\
\hline Jensen-Haise & 0,78 & 0,88 & 0,93 & 0,81 & Muito bom \\
\hline Makkink & 0,72 & 0,84 & 0,89 & 0,75 & Bom \\
\hline Thornthwaite & 0,44 & 0,67 & 0,68 & 0,45 & Mau \\
\hline
\end{tabular}

$r^{2}$ : coeficiente de determinação; r: coeficiente de correlação; d: índice de exatidão; c: índice de concordância.

Fonte: Elaboração própria.

em todos eles, indicando que esses são menos dependentes das condições climáticas da área de estudo em comparação aos métodos que são baseados na temperatura. Percebe-se também que o desempenho dos modelos é diferente dependendo da escala de tempo utilizada, e alguns métodos se adaptam melhor na escala mensal do que na escala diária.

\section{CONCLUSÕES}

Com base na utilização de diferentes métodos indiretos de estimativa de evapotranspiração e comparação com o método de referência Penman-Monteith, definido pela FAO, e considerando as condições climáticas da área de estudo, pode-se chegar às seguintes conclusões:

1. Entre todos os métodos analisados, o método de Blaney-Criddle foi o que apresentou as melhores estimativas da evapotranspiração de referência nas escalas tanto mensal quanto diária;

2. Entre os métodos baseados na radiação solar, o que teve as melhores estimativas é o método de Jensen-Haise nas escalas tanto mensal quanto diária;

3. Na impossibilidade do uso do método de referência, dada a falta de algumas variáveis de entrada, os métodos mais recomendados para a região de estudo são o de Blaney-Criddle e o de Jensen-Haise, que obtiveram desempenho classificado como "ótimo" em escala mensal;

4. Os métodos com os piores desempenhos, e por isso a sua utilização não é recomendada para o município de estudo, foram os de Thornthwaite e Camargo.

\section{CONTRIBUIÇÃO DOS AUTORES}

Ongaratto, J. M.: conceituação, curadoria de dados, análise formal, metodologia, escrita, primeira redação. Bortolin, T.A.: revisão e edição, supervisão.

\section{REFERÊNCIAS}

ALLEN, R.G.; PERREIRA, L.S.D; SMITH, M. Crop evaporation (irrigation and drainage paper 56). Roma: FAO, 1998. $301 \mathrm{p}$

ALMEIDA, B.M.; ARAÚJO, E.M.; JUNIOR, E.G.C.; OLIVEIRA, J.B. Comparação de métodos de estimativa da ETo na escala mensal em FortalezaCE. Revista Brasileira de Agricultura Irrigada, [s.I.], n. 2, p. 93-98, 2010. https:// doi.org/10.7127/rbai.v4n200610

BACK, A.J. Desempenho de métodos empíricos baseados na temperatura do ar para estimativa da evapotranspiração de referência em Urussanga. Revista Irriga, v. 13, n. 4, p. 449-466, 2008. https://doi.org/10.15809/ irriga.2008v13n4p449-466

BORGES JUNIOR, J.C.F; ANJOS, R.J.; SILVA, T.J.A.; LIMA, J.R.S; ANDRADE, C.L.T. Métodos de estimativa da evapotranspiração de referência diária para a microrregião de Garanhuns, PE. Revista Brasileira de Engenharia Agrícola e Ambiental, v. 16, n. 4, p.380-390, 2012. https://doi.org/10.1590/ S1415-43662012000400008

BORTOLIN, T.A., SANTOS, L.M., SILVA, A.G., SCHNEIDER, V.E. Desenvolvimento de uma aplicação web para estimativa de evapotranspiração potencial por diferentes métodos. In: Anais do XIV SRHNE - Simpósio De Recursos Hidricos do Nordeste, Maceió, Alagoas, 14 de novembro de 2018.
BORGES, A.C.; MENDIONDO, E.M. Comparação entre equações empíricas para estimativa da evapotranspiração de referência na Bacia do Rio Jacupiranga. Revista brasileira de Engenharia Agrícola e Ambiental, v. 11, n. 3, p. 293-300, 2007. https://doi.org/10.1590/S1415-43662007000300008

BRIXNER, G.F;; SCHOFFEL, E.R.; TONIETTO, J. Determinação da evapotranspiração por diferentes métodos e sua aplicação no índice de seca na campanha gaúcha, Brasil. Revista Brasileira de Fruticultura, v. 36, n. 4, p. 780-793, 2014. https://doi.org/10.1590/0100-2945-381/13

CAMARGO, A.P.; SENTELHAS, P.C. Avaliação do desempenho de diferentes métodos de estimativa da evapotranspiração potencial no estado de São Paulo. Revista Brasileira de Agrometeorologia, v. 5, n. 1, p. 89-97, 1997.

CAMARGO, A.P.; MARIN, F.R.; SENTELHAS, P.C.; PICINI, A.G. Ajuste da equação de Thornthwaite para estimar a evapotranspiração potencial em climas áridos e superúmidos, com base na amplitude térmica diária. Revista Brasileira de Agrometeorologia, v. 7. n. 2, p. 251-257, 1999. Disponível em: http://www.leb.esalq.usp.br/agmfacil/artigos/artigos_sentelhas_1999/1999 RBAgro_7(2)_251-257_ETPThTefetiva.pdf

CAMARGO, A.P.; CAMARGO, M.B.P. Uma revisão analítica da evapotranspiração potencial. Bragantia, v. 59, n. 2, p. 125-137, 2000. https:// doi.org/10.1590/S0006-87052000000200002 
CAVALCANTI JUNIOR, E.G.; ALMEIDA, B.M; OLIVEIRA, A.D.; SOBRINHO, J.E.; ARAÚJO, E.M.; VIEIRA, R.Y. Estimativa da evapotranspiração de referência para a cidade de Mossoró-RN. Revista Brasileira de Agricultura Irrigada, v. 4, n. 2, p. 87-92, 2010. https://doi.org/10.7127/rbai.v4n200110

CONCEIÇÃO, M.A.F;; MANDELLI, F. Comparação entre métodos de estimativa da evapotranspiração de referência em Bento Gonçalves, RS. Revista Brasileira de Agrometeorologia, v. 13, n. 2, p. 303-307, 2005. ISSN 0104-1347.

CRUZ, J.T. Avaliação de métodos de estimativa de evapotranspiração para Brasília - DF. 2016. 45 p. Monografia (Graduação em Agronomia). Universidade de Brasília, Brasília.

FERNANDES, L.C. Avaliação de diversas equações empíricas de evapotranspiração. Estudo de caso: Campos dos Goytacazes e Ilha do Fundão-RJ. 2006. 142 f. Dissertação (Mestrado em Engenharia Civil) - Curso de Engenharia Civil, Universidade Federal do Rio de Janeiro, Rio de Janeiro.

GONÇALVES, F.M.; FEITOSA, H.O.; CARVALHO, C.M.; GOMES FILHO, R.R.; VALNIR JÚNIOR, M. Comparação de métodos da estimativa da evapotranspiração de referência para o município de Sobral - CE. Revista Brasileira de Agricultura Irrigada, v. 3, n. 2, p. 71-77, 2009. https://doi. org/10.7127/rbai.v3n200016

GOTARDO, J.T.; RODRIGUES, L.N.; GOMES, B.M. Comparison of methods for estimating reference evapotranspiration: an approach to the management of water resources within an experimental basin in the Brazilian. Revista Engenharia Agrícola, v. 36, n. 6, p. 1016-1026, 2016. https:// doi.org/10.1590/1809-4430-Eng.Agric.v36n6p1016-1026/2016

JUNG, L.H.; BISCAVO, G.A.; OLIVEIRA, G.Q.; ALVES, M.A.; GIACON, G.M. Estimativa da evapotranspiração de referência em uma região do Alto Pantanal. Magistra, v. 28, n. 2, p. 168-177, 2016. Disponível em: https:// magistraonline.ufrb.edu.br/index.php/magistra/article/view/265

LIMA JUNIOR, J.C.; ARRAES, F.D.D.; OLIVEIRA, J.B.; NASCIMENTO, F.A.L; MACÊDO, K.G. Parametrização da equação de Hargreaves e Samani para estimativa da evapotranspiração de referência no Estado do Ceará, Brasil. Revista Ciência Agronômica, v. 47, n. 3, p. 447-454, 2016. Disponível em: http://ccarevista.ufc.br/seer/index.php/ccarevista/article/view/4018

OLIVEIRA, L.M.M. Avaliação da evapotranspiração de referencia (ETo) na bacia experimental do riacho gameleira-PE utilizando lisimetro de pesagem hidráulica e métodos indiretos. 2007. 110 f. Dissertação (Mestrado em Tecnologia Ambiental e Recursos Hídricos). Universidade Federal de Pernambuco, Recife.

OLIVEIRA, L.M.M.; MONTENEGRO, S.M.G.L.; AZEVEDO, J.R.G.; SANTOS, F.X. Evapotranspiração de referência na bacia experimental do riacho Gameleira, PE, utilizando-se lisímetro e métodos indiretos. Revista Brasileira de Ciências Agrárias, v. 3, n. 1, p. 58-67, 2008. https://doi.org/0.5039/agraria. v3ila250

PAIVA, C.M.S.; PINHEIRO, A.S. Avaliação de métodos de estimativa da evapotranspiração de referência para fins de manejo da irrigação Anuário do Instituto de Geociências, v. 39, n. 1, p. 42-51, 2016. http://doi. org/10.11137/2016_1_42_51

PALARETTI, L.F.; MANTOVANI, E.C.; SEDIYAMA, G.C. Comparação entre método de estimativa de evapotranspiração de referência (ETo) em regiões citrícolas Paulistas. Revista Engenharia Agrícola, v. 34, n. 1, p. 38-47, 2014. https://doi.org/10.1590/S0100-69162014000100005

PEREIRA, D.P.; YANAGI, S.N.M.; MELLO, C.R.; SILVA, A.M.; SILVA, L.A. Desempenho de métodos de estimativa da evapotranspiração de referência para a região da Serra da Mantiqueira, MG. Ciência Rural, v. 39, n. 9. p. 2488-2493, 2009. https://doi.org/10.1590/S0103-84782009000900016

PILAU, F.G.; BATTISTI, R.; SOMAVILLA, L.; RIGHI, E.V. Desempenho de métodos de estimativa da evapotranspiração de referência nas localidades de Frederico Westphalen e Palmeira das Missões, RS. Ciência Rural, v. 42, n. 2, p. 283-290, 2012. https://doi.org/10.1590/S0103-84782012000200016

SANCHES, F.M.; XIMENES, A.R.; CORADI, P.C.; ROQUE, C.G.; CUNHA, F.F. Estimativa da evapotranspiração de referência na região norte do Brasil. Revista de Ciências Agroambientais, v. 13, n. 2, p. 19-31, 2015. https://doi. org/10.5327/rcaa.v13i2.1179

SILVEIRA, P.M.; STONE, L.F. Manejo da irrigação do feijoeiro: Uso do tensiômetro e avaliação do desempenho do pivô central. Goiânia: Embrapa Arroz e Feijão, 1994. 46 p.

VAREJÃO-SILVA, M.A. Meteorologia e Climatologia. Recife: 2006. Versão Digital, $443 p$

WILLMOTT, C.J.; ACKLESON, S.G.; DAVIS, R.E.; FEDDEMA, J.J.; KLINK, K.M.; LEGATES, D.R.; O'DONNELL, J.; ROWE, C.M. Statistics for evaluation and comparison of models. Journal of Geophysical Research, v. 90, n. C5, p. 8995-9005, 1985. https://doi.org/10.1029/JC090iC05p08995 